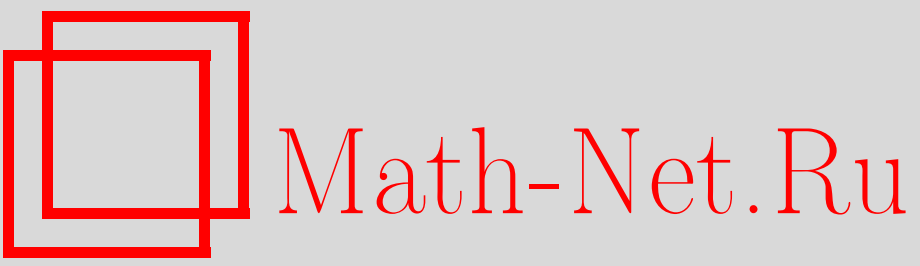

L. Nguyen-Ngoc, Limiting laws and penalization of certain Lévy processes by a function of their maximum, Теория вероятн. и ее примен., 2010, том 55, выпуск $3,530-547$

DOI: https://doi.org/10.4213/tvp4240

Использование Общероссийского математического портала Math-Net.Ru подразумевает, что вы прочитали и согласны с пользовательским соглашением

http://www. mathnet.ru/rus/agreement

Параметры загрузки:

IP : 54.157 .27 .8

26 апреля 2023 г., 17:55:31 


\title{
LIMITING LAWS AND PENALIZATION OF CERTAIN LÉVY PROCESSES BY A FUNCTION OF THEIR MAXIMUM
}

\begin{abstract}
Изучается ряд примеров штрафных функционалов от распределений некоторых спектрально отрицательных процессов Леви $X$, включая процесс максимума и отраженный процесс. В частности, приводятся траекторные разложения относительно штрафного распределения. Система Леви процесса $X$ относительно штрафного распределения вычисляется в явном виде.
\end{abstract}

Ключевые слова и фразы: процессы Леви, мартингалы, траекторные разложения.

1. Introduction. In some recent papers [10]-[12] the authors studied penalizations of Brownian motion by a function of its maximum. More precisely, if $P$ is the Wiener measure on the Wiener space $C\left(\mathbf{R}_{+}, \mathbf{R}\right), X$ the canonical process and $S_{t}=\sup _{s \leqslant t} X_{s}$, they investigated the limit

$$
Q(A)=\lim _{t \rightarrow \infty} \frac{\mathbf{E}\left[1_{A} f\left(X_{t}, S_{t}\right)\right]}{\mathbf{E}\left[f\left(X_{t}, S_{t}\right)\right]}, \quad A \in \mathscr{F}_{s}
$$

for suitable functions $f$. In [11] a «meta-theorem» is also presented, which gives sufficient conditions for the existence of the limit above, in a more general context than Brownian motion; however the only case examplified in the works cited above is that of Brownian motion.

In the present paper, we develop similar results for Lévy processes. Specifically we show that the limit (1.1) exists,

(a) when $f(x, s) \equiv f(s)$ with mild regularity assumptions on $f$, if $X$ is a spectrally negative Lévy process whose inverse Laplace exponent has regular variation at 0 with index $\alpha \in(0,1)$; the resulting law is related to the generalized Azéma-Yor martingale discussed in [9];

(b) when $f(x, s)=g(s) e^{\lambda(s-x)}$ for adequate $\lambda \in \mathbf{R}$ and $g$; this latter case is related to the generalized Kennedy martingales (see [9]);

*Département de Mathématiques, Université Paris 12, 61 avenue du Général de Gaulle 94000 Créteil cedex, France; e-mail: laurent.nguyenngoc@gmail.com 
In the Brownian case, the relationship with the usual Azéma-Yor and Kennedy martingales was noticed in [10]-[12].

In each case we study the limiting law $Q$. In particular we show that it corresponds at least intuitively to a weak conditioning of $S_{\infty}$ as developed in [1], [2], [8]. In the case (b), it is actually a weak conditioning after a preliminary change of measure (Esscher transform), but in the case (a) it should rather be interpreted as a singular conditioning (a limiting procedure is necessary).

We also give another description of the disintegrated measure $Q\left(\cdot \mid S_{\infty}=y\right)(y>0)$ by means of a path decomposition at time $g$, the last time $X$ attains $S_{\infty}$, i.e., $g=\sup \left\{t: X_{t}=S_{\infty}\right.$ or $\left.X_{t-}=S_{\infty}\right\}$. Heuristically, these path decompositions read as

- In case (a), the processes $X^{g}=\left(X_{t}, t<g\right)$ and $\left(X_{g+t}, t \geqslant 0\right)$ are independent, the former has the law of $\left(X_{t}, t<T_{y}\right)$ under $P$ (here $\left.T_{y}=\inf \left\{t: X_{t}=y\right\}\right)$ and the latter has the law of $X$ conditioned to stay below $y$;

- In case (b), $X^{g}$ and $X_{g+}$. are independent, the former has the law of $\left(X_{t}, t<T_{y}\right)$ under $\mathbf{P}^{(+)}$and the latter has the law of $X$ conditioned to stay below $y$ under $\mathbf{P}^{(-)} ; \mathbf{P}^{( \pm)}$are Esscher transforms of the original law $\mathbf{P}$ that are naturally associated with the generalized Kennedy martingales.

The paper is organized as follows. Some notation and basic properties of spectrally negative Lévy processes are collected in Section 2. In Section 3 we deal with case (a). Brownian motion falls into this category with $\alpha=$ $1 / 2$. In [10]-[12] the authors often rely on explicit calculations which can be carried out in the Brownian case; in general, such calculations are not feasible, but Tauberian theory allows us to dispense with them.

Case (b) is studied in the final Section 4.

2. Notation and preliminaries. We denote by $\Omega$ the canonical Skorokhod space of càdlàg functions $[0, \infty) \rightarrow \mathbf{R}$. It is endowed with Skorokhod's topology and Borel $\sigma$-field $\mathscr{F}$. The process $X$ is the coordinate process over $\Omega$, i.e., $X_{t}(\omega)=\omega(t)$, and $\mathscr{F}_{t}$ is the associated canonical filtration. The shift operators $\theta_{s}, s \geqslant 0$, are defined by $\theta_{s}(X)=\left(X_{s+t}-X_{s}, t \geqslant 0\right)$. For any random time $T$, we shall denote by $X^{T}$ the process killed at time $T$, namely $X^{T}=\left(X_{t}, t<T\right)$.

We consider $\mathbf{P}$ a probability law on $\Omega$ under which $X$ is a spectrally negative Lévy process started at 0 ; we call $\psi$ the corresponding Laplace exponent of $X: \mathbf{E} e^{\lambda X_{t}}=e^{t \psi(\lambda)}$. Recall that if $\Phi(0)=\sup \{\lambda: \psi(\lambda)=0\}$, then one can define $\Phi=\psi^{-1}$ on $[0, \infty)$ (see [4] for details). 
The running supremum of $X$ will be denoted by $S_{t}$ : $S_{t}=\sup _{s \leqslant t} X_{s}$. Similarly, $I_{t}=\inf _{s \leqslant t} X_{s}$. Then, $S_{t}=S_{u} \vee\left(X_{u}+S_{t-u} \circ \theta_{u}\right), u<t$, where $S_{u}$ and $X_{u}$ are $\mathscr{F}_{u}$-measurable while $S_{t-u} \circ \theta_{u}$ is $\mathbf{P}$-independent of $\mathscr{F}_{u}$. For $x>0$, we denote by $T_{x}$ the first hitting time of $x: T_{x}=\inf \left\{t: X_{t}>x\right\}$; in the case of a spectrally negative Lévy process, we have $X_{T_{x}}=x$ a.s., so $T_{x}=\inf \left\{t: X_{t}=x\right\}$.

Lastly, recall the following basic property: if $\tau$ is an exponential random variable with parameter $q$, independent of $X$, then $S_{\tau}$ has exponential distribution with parameter $\Phi(q)$ :

$$
\mathbf{P}\left[S_{\tau}>x\right]=e^{-x \Phi(q)}, \quad x>0 .
$$

3. Penalization by a function of $S$. In this section, we consider the limit behavior, as $t \rightarrow \infty$, of the laws $\mathbf{Q}_{t}$ defined by

$$
\mathbf{Q}_{t}(\Lambda)=\frac{\mathbf{E}\left[\xi\left(S_{t}\right) 1_{\Lambda}\right]}{\mathbf{E}\left[\xi\left(S_{t}\right)\right]}, \quad \Lambda \in \mathscr{F}_{t},
$$

for some suitably chosen function $\xi$.

In all this section, we shall make the following standing assumption.

Assumption 3.1. The inverse Lévy exponent $\Phi$ has regular variation at 0 , with index $\alpha \in(0,1)$.

Thus, there is a function $\lambda$ slowly varying at 0 such that $\Phi(q) \sim q^{\alpha} \lambda(q)$ as $q \rightarrow 0$.

Note that Assumption 3.1 implies that $X$ is a martingale. Indeed, under this assumption, we have $\Phi(0)=0$ and $\Phi^{\prime}(0+)=+\infty$; it is well known that these conditions imply that $X$ oscillates, that is, $X$ is a martingale.

Intuitively, Assumption 3.1 means that $X$ approaches large levels in a way similar to a stable process with index $1 / \alpha$. Of course the Brownian case treated in [10]-[12] corresponds to $\alpha=1 / 2$.

Now, let $\xi: \mathbf{R}_{+} \rightarrow \mathbf{R}_{+}$be an integrable function, such that $\int \xi(x) d x=1$ (thus, $\xi$ is a probability density over $\mathbf{R}_{+}$). We assume moreover that $a \leqslant$ $\xi \leqslant b$ for some $0<a<b$. This latter assumption is certainly not necessary but it shall simplify matters technically.

We denote by $\Xi$ the associated cumulative distribution function:

$$
\Xi(x)=\int_{0}^{x} \xi(y) d y .
$$

Recall the following result, which generalizes the Azéma-Yor martingales.

Proposition 3.1 [9]. The process

$$
M_{t}^{\xi}=1-\Xi\left(S_{t}\right)+\left(S_{t}-X_{t}\right) \xi\left(S_{t}\right)
$$

is a local martingale. 
The process $M^{\xi}$ is a true martingale as soon as $\xi$ is bounded and $I_{t}$ is integrable. Indeed, we have then

$$
\sup _{s \leqslant t}\left|M_{s}^{\xi}\right| \leqslant 1+\left(S_{t}-I_{t}\right)\|\xi\|_{\infty}
$$

so that $M^{\xi}$ is a class (D) local martingale.

$\mathrm{Ex}$ a $\mathrm{m} \mathrm{ple} \mathrm{3.1.} \mathrm{If} X$ is a stable process with index $\beta$, then

$$
\mathbf{P}\left[\left|I_{t}\right|>x\right] \sim k x^{-\beta}, \quad x \rightarrow+\infty
$$

(see [4, p. 221]), where $k>0$ is a constant. Hence in this case, $M^{\xi}$ is a true martingale as soon as $\beta>1$ and $\xi$ is bounded.

3.1. The penalized law and a pathwise description. From now on, we assume that $M^{\xi}$ is a true martingale. We then have the following theorem.

Theorem 3.1. For $u \geqslant 0$ and $\Lambda \in \mathscr{F}_{u}$, the limit

$$
\lim _{t \rightarrow \infty} \frac{\mathbf{E}\left[\xi\left(S_{t}\right) 1_{\Lambda}\right]}{\mathbf{E}\left[\xi\left(S_{t}\right)\right]}
$$

exists and is equal to $\mathbf{E}\left[M_{u}^{\xi} 1_{\Lambda}\right]$.

The proof of the theorem relies on the following lemma.

Lemma 3.1. Let $l(t)=\lambda(1 / t)$. For $x \in \mathbf{R}$ and $s \geqslant 0 \vee x$, we have

$$
\mathbf{E}\left[\xi\left(s \vee\left(x+S_{t}\right)\right)\right] \sim \frac{l(t)}{t^{\alpha}}((s-x) \xi(s)+1-\Xi(s)), \quad t \rightarrow \infty .
$$

$\mathrm{P} \mathrm{r}$ o o f. Let $\tau$ be an exponential variable with parameter $q$, independent of $X$. We have

$$
\mathbf{E}\left[\xi\left(s \vee\left(x+S_{\tau}\right)\right)\right]=\xi(s)\left(1-e^{-(s-x) \Phi(q)}\right)+\Phi(q) e^{x \Phi(q)} \int_{s}^{\infty} e^{-y \Phi(q)} \xi(y) d y .
$$

Letting $q \downarrow 0$, we obtain

$\int_{0}^{\infty} e^{-q t} \mathbf{E}\left[\xi\left(s \vee\left(x+S_{t}\right)\right)\right] d t \sim q^{\alpha-1} \lambda(q)((s-x) \xi(s)+1-\Xi(s)), \quad q \rightarrow 0$.

The result then follows from a Tauberian theorem (see [6]).

$\mathrm{P}$ r o of of $\mathrm{Th}$ e or e $\mathrm{m} 3.1$. For $t \geqslant u$, we have

$$
\mathbf{Q}_{t}(\Lambda)=\mathbf{E}\left(1_{\Lambda} \frac{\mathbf{E}\left[\xi\left(s \vee\left(x+S_{t-u}\right)\right)\right]}{\mathbf{E}\left[\xi\left(S_{t}\right)\right]}\right)
$$

for $s=S_{u}$ and $x=X_{u}$. Using Lemma 3.1 and the dominated convergence theorem yields the theorem. 
Theorem 3.1 implies that there exists a probability measure $\mathbf{Q}$ on $(\Omega, \mathscr{F})$ such that, for each $t \geqslant 0$,

$$
\left.\mathbf{Q}\right|_{\mathscr{F}_{t}}=\left.M_{t}^{\xi} \mathbf{P}\right|_{\mathscr{F}_{t}} .
$$

The rest of this section is devoted to the study and description of this law $\mathbf{Q}$.

First, we note that although $\mathbf{Q}$ is locally absolutely continuous with respect to $\mathbf{P}$, these two measures are singular. Indeed, since the process $X$ oscillates, we have $\mathbf{P}\left(S_{\infty}=\infty\right)=1$ while the following proposition implies that $\mathbf{Q}\left(S_{\infty}<\infty\right)=1$.

Proposition 3.2. The law of $S_{\infty}$ under $\mathbf{Q}$ is given by

$$
\mathbf{Q}\left[S_{\infty} \leqslant x\right]=\Xi(x) .
$$

P r o o f. Let $\tau$ be an exponential variable with parameter $q$, independent of $X$. We have, since $S_{\tau}-X_{\tau}$ is independent of $S_{\tau}$ (see [4]):

$$
\begin{aligned}
\mathbf{Q}\left[S_{\tau} \leqslant x\right] & =\mathbf{E}\left[M_{\tau}^{\xi} 1_{S_{\tau} \leqslant x}\right] \\
& =\mathbf{E}\left[\left(1-\Xi\left(S_{\tau}\right)+\left(S_{\tau}-X_{\tau}\right) \xi\left(S_{\tau}\right)\right) 1_{S_{\tau} \leqslant x}\right] \\
& =\mathbf{P}\left[S_{\tau} \leqslant x\right]-\mathbf{E}\left[\Xi\left(S_{\tau}\right) 1_{S_{\tau} \leqslant x}\right]+\mathbf{E}\left[S_{\tau}-X_{\tau}\right] \mathbf{E}\left[\xi\left(S_{\tau}\right) 1_{S_{\tau} \leqslant x}\right] \\
& =1-e^{-x \Phi(q)}(1-\Xi(x)) .
\end{aligned}
$$

By letting $q \downarrow 0$ we obtain the announced result.

In order to complete the description of $\mathbf{Q}$, we now wish to describe its disintegration with respect to $S_{\infty}$. Our first result in this direction is a path decomposition under $\mathbf{Q}$. It will involve the law $\mathbf{P}^{\downarrow}$ of $\ll X$ conditioned to stay negative». We recall here briefly the construction of [5] for the reader's convenience.

First, for $x \in \mathbf{R}$ let $\mathbf{P}_{x}$ be the law of $x+X$ under $\mathbf{P}$; the family $\left(\mathbf{P}_{x}, x \in \mathbf{R}\right)$ is then strongly Markovian. For $x<0$, it is shown in [5] that the limit

$$
\mathbf{P}_{x}^{\downarrow}(\Lambda)=\lim _{q \rightarrow 0} \mathbf{P}_{x}\left(\Lambda, t<\tau, S_{\tau} \leqslant 0\right), \quad t>0, \quad \Lambda \in \mathscr{F}_{t},
$$

exists, where $\tau$ denotes an exponential variable with parameter $q$, independent of $X$. Moreover, for $t \geqslant 0$ and $\Lambda \in \mathscr{F}_{t}$,

$$
\mathbf{P}_{x}^{\downarrow}(\Lambda)=\frac{1}{x} \mathbf{E}_{x}\left(X_{t} 1_{\Lambda} 1_{S_{t} \leqslant 0}\right) .
$$

Now, let $g=\sup \left\{t>0: X_{t}=S_{\infty}\right.$ or $\left.X_{t-}=S_{\infty}\right\}$. Since under $\mathbf{P}_{x}^{\downarrow}, X_{t} \rightarrow-\infty$ a.s. as $t \rightarrow \infty$, we have $g<\infty \mathbf{P}_{x}^{\downarrow}$-a.s. The law $\mathbf{P}^{\downarrow}$ is then defined as the law of the process $\left(X_{g+t}-X_{g-}, t \geqslant 0\right)$. When $X$ has unbounded variation, one also has $\mathbf{P}^{\downarrow}=\lim _{x \uparrow 0} \mathbf{P}_{x}^{\downarrow}$. 
We are now ready to give the path decomposition of $X$ at time $g$ under Q. In [11] it is proved in the Brownian case by means of the theory of enlargement of filtrations. Here, we use excursion theory instead.

Recall that $S-X$ is a Feller process and that $S$ is a local time at 0 ; then, the process of first hitting times

$$
T_{x}=\inf \left\{t: X_{t}>x\right\}=\inf \left\{t: X_{t}=x\right\}, \quad x \geqslant 0,
$$

is the inverse local time. We denote by $\mathscr{L}$ the set of left-end points $l$ of the excursion intervals $(l, d)$ of $S-X$ away from 0 , and by $n$ the Itô measure associated with the excursion process $e=\left(e_{l}, l \in \mathscr{L}\right)$ with $e_{l}(s)=S_{l}-X_{l+s}$, $s<d-l$.

Theorem 3.2. Under $\mathbf{Q}$, conditionally on $S_{\infty}=y$, the processes $\left(X_{t}, t<g\right)$ and $\left(X_{g+t}, t \geqslant 0\right)$ are independent. Moreover,

(a) The law of $\left(X_{t}, t<g\right)$ under $\mathbf{Q}\left(\cdot \mid S_{\infty}=y\right)$ is the law of $\left(X_{t}, t<T_{y}\right)$ under $\mathbf{P}$;

(b) the law of $\left(X_{g+t}, t>0\right)$ under $\mathbf{Q}\left(\cdot \mid S_{\infty}=y\right)$ is characterized by

$$
\mathbf{Q}\left(K\left(X_{g+t}, t \geqslant 0\right) \mid S_{\infty}=y\right)=\mathbf{E}^{\downarrow}\left[K\left(y+X_{t}, t \geqslant 0\right)\right]
$$

for all measurable and bounded functionals $K$.

P r o o f. Let $\tau$ be an exponential variable with parameter $q$, independent of $X$. We denote by $g_{\tau}$ the last time $X$ reaches its supremum before time $\tau$, that is

$$
g_{\tau}=\sup \left\{t \leqslant \tau: X_{t}=S_{t}\right\} .
$$

The killed process $X^{\tau}=\left(X_{s}, s<\tau\right)$ has a pre-maximum process which we denote by $\overleftarrow{X^{\tau}}=\left(X_{s}, s<g_{\tau}\right)$ and a post-maximum process denoted by $\overrightarrow{X^{\tau}}=\left(X_{g_{\tau}+s}, s<\tau-g_{\tau}\right)$.

Let $H$ and $K$ be two measurable, bounded functionals on $\Omega$. We have

$$
\begin{aligned}
\mathbf{Q} & \left(H\left(\overleftarrow{X^{\tau}}\right) K\left(\overrightarrow{X^{\tau}}\right)\right)=\mathbf{E}\left[M_{\tau}^{\xi} H\left(\overleftarrow{X^{\tau}}\right) K\left(\overrightarrow{X^{\tau}}\right)\right] \\
\quad & =\mathbf{E}\left[\left(1-\Xi\left(S_{\tau}\right)\right) H\left(X^{\tau}\right) K\left(\overrightarrow{X^{\tau}}\right)\right]+\mathbf{E}\left[\left(S_{\tau}-X_{\tau}\right) \xi\left(S_{\tau}\right) H\left(\overleftarrow{X^{\tau}}\right) K\left(\overrightarrow{X^{\tau}}\right)\right]
\end{aligned}
$$

Regarding the former term,

$$
\begin{aligned}
\mathbf{E} \Xi\left(S_{\tau}\right) & =\int_{0}^{\infty} \Phi(q) e^{-\Phi(q) x} \int_{0}^{x} \xi(y) d y d x=\int_{0}^{\infty} \xi(y) \int_{y}^{\infty} \Phi(q) e^{-\Phi(q) x} d x d y \\
& =\int_{0}^{\infty} \xi(y) e^{-\Phi(q) y} d y
\end{aligned}
$$

and this tends to 1 when $q \rightarrow 0$. 
Now, note that for all $t$ in an excursion interval $(l, d)$, we have $S_{t}=S_{l}$ and $S_{t}-X_{t}=e_{l}(t-l)$, where $e_{l}$ is the corresponding excursion, so that $M_{t}^{\xi}=1-\Xi\left(S_{l}\right)+e_{l}(t-l) \xi\left(S_{l}\right)$. Also, for $t \in(l, d)$,

$$
\overleftarrow{X^{t}}=X^{l}=\left(X_{s}, s<l\right) \quad \text { and } \quad \overrightarrow{X^{t}}=\left(X_{l+s}, s<t-l\right)
$$

Hence we have

$$
\begin{aligned}
& \mathbf{E}\left[\left(S_{\tau}-X_{\tau}\right) \xi\left(S_{\tau}\right) H\left(\overleftarrow{X^{\tau}}\right) K\left(\overrightarrow{X^{\tau}}\right)\right] \\
& \quad=\mathbf{E}\left[\sum_{l \in \mathscr{L}} \int_{l}^{d} q e^{-q t} e_{l}(t-l) \xi\left(S_{l}\right) H\left(X^{l}\right) K\left(X_{l+s}, s<t-l\right) d t\right] .
\end{aligned}
$$

The compensation formula entails that the foregoing equals

$$
\mathbf{E}\left(\int_{0}^{\infty} e^{-q T_{l}} \xi(l) H\left(X^{T_{l}}\right) \cdot q n\left(\int_{0}^{\zeta} e^{-q t} \varepsilon(t) K(l-\varepsilon(s), s<t) d t\right) d l\right),
$$

where $\varepsilon(t)$ is a generic excursion. Recall that for any measurable functional $F$,

$$
n\left(\int_{0}^{\zeta} e^{-q t} F(\varepsilon(s), s<t) d t\right)=\mathbf{E}^{\downarrow}\left(\int_{0}^{\infty} e^{-q t} \frac{1}{\left|X_{t}\right|} F\left(-X_{s}, s<t\right) d t\right)
$$

(see, e.g., [5, end of the proof of Theorem 4, p. 16]. Hence, we have

$$
q n\left(\int_{0}^{\zeta} e^{-q t} \varepsilon(t) K(l-\varepsilon(s), s<t) d t\right)=\mathbf{E}^{\downarrow}\left(\int_{0}^{\infty} q e^{-q t} K\left(l+X_{s}, s<t\right)\right)
$$

so that, as $q \downarrow 0$,

$$
q n\left(\int_{0}^{\zeta} e^{-q t} \varepsilon(t) K(l-\varepsilon(s), s<t) d t\right) \longrightarrow \mathbf{E}^{\downarrow} K\left(l+X_{t}, t>0\right) .
$$

Moreover,

$$
\mathbf{E}\left[\xi(l) H\left(X^{T_{l}}\right) e^{-q T_{l}}\right] \longrightarrow \mathbf{E}\left[\xi(l) H\left(X^{T_{l}}\right)\right], \quad q \rightarrow 0,
$$

and since $\xi \in L^{1}\left(\mathbf{R}_{+}\right)$, we can use the dominated convergence theorem to obtain that

$\mathbf{Q}\left(H\left(\overleftarrow{X^{\tau}}\right) K\left(\overrightarrow{X^{\tau}}\right)\right) \longrightarrow \int_{0}^{\infty} \xi(l) \mathbf{E}\left(H\left(X^{T_{l}}\right)\right) \mathbf{E}^{\downarrow}\left(K\left(l+X_{t}, t>0\right)\right) d l, \quad q \rightarrow 0$

and the proof is complete.

As we have just seen, the law of $S_{\infty}$ under $\mathbf{Q}$ has density $\xi$; it is now natural to ask whether the measure $\mathbf{Q}$ may be constructed from $\mathbf{P}$ in a maybe more natural manner, by «conditioning $S_{\infty}$ to have the law $\xi »$. In [1], [2], [8], 
the following problem was studied: for fixed $T$, given an $\mathscr{F}_{T}$-measurable functional $F$ with values in a space $E$ and a probability law $\mu$ on $E$, construct a probability measure $\mathbf{Q}$ such that $\mathbf{Q} \circ F^{-1}=\mu$. However the setting was different, especially as it is assumed in these works that $\mu \ll \mathbf{P} \circ F^{-1}$. Nevertheless, our next result shows that $\mathbf{Q}$ can indeed be obtained in a similar way.

Proposition 3.3. Assume that for $t \geqslant 0, S_{t}$ has a density $p_{t}$ with respect to Lebesgue measure. Then:

(a) For every $y>0, s \geqslant 0$ and $\Lambda \in \mathscr{F}_{s}$, the limit

$$
Q^{(y)}(\Lambda)=\lim _{t \rightarrow \infty} \mathbf{P}\left[\Lambda \mid S_{t}=y\right]
$$

is well defined and moreover

$$
Q^{(y)}(\Lambda)=p_{s}(y) \mathbf{E}\left[\left(y-X_{s}\right) 1_{\Lambda} \mid S_{s}=y\right]+\mathbf{P}\left[\Lambda, S_{s} \leqslant y\right] ;
$$

(b) the law $\mathbf{Q}$ is given by

$$
\mathbf{Q}=\int_{0}^{\infty} Q^{(y)} \xi(y) d y .
$$

The proof of the proposition relies on the following lemma.

Lemma 3.2. Assume that $S_{t}$ has a density $p_{t}$ for all $t \geqslant 0$. Then, (a) For all fixed $x>0$ :

$$
p_{t}(x) \sim \frac{l(t)}{t^{\alpha}}, \quad t \rightarrow \infty
$$

moreover,

$$
\mathbf{P}\left(S_{t}<x\right) \sim x \frac{l(t)}{t^{\alpha}}, \quad t \rightarrow \infty ;
$$

(b) if $y>0,0 \leqslant u<t$, and $\Lambda \in \mathscr{F}_{u}$, then,

$$
\begin{aligned}
\mathbf{P}\left(\Lambda \mid S_{t}=y\right)= & \frac{p_{u}(y)}{p_{t}(y)} \mathbf{E}\left(1_{\Lambda} h\left(t-u, y-X_{u}\right) \mid S_{u}=y\right) \\
& +\frac{1}{p_{t}(y)} \mathbf{E}\left(1_{\Lambda} 1_{S_{u}<y} p_{t-u}\left(y-X_{u}\right)\right) .
\end{aligned}
$$

P r o o f. (a) Let $\tau$ be an exponential variable with parameter $q>0$, independent of $X$. Then, $\mathbf{P}\left(S_{\tau} \in(a, b]\right)=e^{-\Phi(q) a}-e^{-\Phi(q) b}$ and this is equivalent, for $q \downarrow 0$, to $\lambda(q) q^{\alpha}(b-a)$. We deduce from a Tauberian theorem that $\mathbf{P}\left(S_{\tau} \in(a, b]\right) \sim l(t) t^{-\alpha}(b-a)$ as $t \rightarrow \infty$. This implies (3.10) and (3.11).

(b) Let $f$ be a positive Borel function on $\mathbf{R}_{+}$. We first note that $\mathbf{E}\left(1_{\Lambda} f\left(S_{t}\right)\right)=\mathbf{E}\left(1_{\Lambda} f\left(S_{u} \vee\left(X_{u}+S_{t-u} \circ \theta_{u}\right)\right)\right)$. We have

$$
\begin{aligned}
& \mathbf{E}\left(1_{\Lambda} f\left(S_{t}\right)\right)=\mathbf{E}\left(1_{\Lambda} f\left(S_{u} \vee\left(X_{u}+S_{t-u} \circ \theta_{u}\right)\right)\right) \\
& \quad=\mathbf{E}\left(1_{\Lambda} f\left(S_{u}\right) 1_{S_{u} \geqslant X_{u}+S_{t-u} \circ \theta_{u}}\right)+\mathbf{E}\left(1_{\Lambda} f\left(X_{u}+S_{t-u} \circ \theta_{u}\right) 1_{S_{u}<X_{u}+S_{t-u} \circ \theta_{u}}\right) .
\end{aligned}
$$


The first term can be expressed as

$$
\begin{gathered}
\mathbf{E}\left(1_{\Lambda} f\left(S_{u}\right) 1_{S_{u} \geqslant X_{u}+S_{t-u} \circ \theta_{u}}\right)=\mathbf{E}\left(1_{\Lambda} f\left(S_{u}\right) h\left(t-u, S_{u}-X_{u}\right)\right) \\
=\int_{0}^{\infty} p_{u}(y) f(y) \mathbf{E}\left(1_{\Lambda} h\left(t-u, y-X_{u}\right) \mid S_{u}=y\right) d y .
\end{gathered}
$$

The second term can be written in the following form:

$$
\begin{aligned}
\mathbf{E}\left(1_{\Lambda}\right. & \left.f\left(X_{u}+S_{t-u} \circ \theta_{u}\right) 1_{S_{u}<X_{u}+S_{t-u} \circ \theta_{u}}\right) \\
& =\mathbf{E}\left(1_{\Lambda} \int_{S-u-X_{u}}^{\infty} f\left(X_{u}+x\right) p_{t-u}(x) d x\right) \\
& =\mathbf{E}\left(1_{\Lambda} \int_{S_{u}}^{\infty} f(y) p_{t-u}\left(y-X_{u}\right) d y\right) \\
& =\int_{0}^{\infty} f(y) \mathbf{E}\left(1_{\Lambda} 1_{S_{u}<y} p_{t-u}\left(y-X_{u}\right)\right) d y .
\end{aligned}
$$

On the other hand,

$$
\mathbf{E}\left(1_{\Lambda} f\left(S_{t}\right)\right)=\int_{0}^{\infty} f(y) p_{t}(y) \mathbf{P}\left(\Lambda \mid S_{t}=y\right) d y
$$

so the proof is complete.

Pro of of Proposition 3.3. From the above lemma, we deduce that, for $t \rightarrow \infty$,

$$
\begin{aligned}
\mathbf{P}\left(\Lambda \mid S_{t}=y\right) \sim & p_{u}(y) \frac{l(t-u)}{l(t)} \frac{t^{\alpha}}{(t-u)^{\alpha}} \mathbf{E}\left(1_{\Lambda}\left(y-X_{u}\right) \mid S_{u}=y\right) \\
& +\frac{l(t-u)}{l(t)} \frac{t^{\alpha}}{(t-u)^{\alpha}} \mathbf{P}\left(\Lambda, S_{u}<y\right)
\end{aligned}
$$

and this implies that the limit in (3.7) exists and is equal to (3.8).

Now,

$$
\begin{aligned}
\int_{0}^{\infty} Q_{0}^{(y)}(\Lambda) \xi(y) d y= & \int_{0}^{\infty} \xi(y) p_{u}(y) \mathbf{E}\left(1_{\Lambda}\left(y-X_{u}\right) \mid S_{u}=y\right) d y \\
& +\int_{0}^{\infty} \xi(y) \mathbf{P}\left(\Lambda, S_{u}<y\right) d y .
\end{aligned}
$$

The first term is equal to $\mathbf{E}\left(1_{\Lambda} \xi\left(S_{u}\right)\left(S_{u}-X_{u}\right)\right)$, while thanks to the FubiniTonelli theorem, we have for the second term

$$
\int_{0}^{\infty} \xi(y) \mathbf{P}\left(\Lambda, S_{u}<y\right) d y=\mathbf{E}\left(1_{\Lambda}\left(1-\Xi\left(S_{u}\right)\right) .\right.
$$

In summary,

$$
\int_{0}^{\infty} Q_{0}^{(y)}(\Lambda) \xi(y) d y=\mathbf{E}\left(1_{\Lambda} M_{u}^{\xi}\right)=\mathbf{Q}(\Lambda)
$$

as expected. 
3.2. A martingale point of view. In this subsection, we study the law $\mathbf{Q}$ from the standpoint of martingale theory. More precisely, we are interested in understanding the semimartingale decomposition of $X$ under $\mathbf{Q}$.

In the continuous case (i.e., the Brownian case), the Girsanov theorem gives the decomposition of $X$ as a semimartingale: it is Brownian motion with drift $\mu_{t}$ of the form $f\left(X_{t}, S_{t}\right)$.

In our setting, since the continuous martingale part of $X$ is Brownian motion under $\mathbf{P}$, so will it be under $\mathbf{Q}$. Hence we turn our attention to the jumps of $X$. Denote by $\nu$ the Lévy measure of $X$ (under $\mathbf{P}$ ).

$\mathrm{R}$ e $\mathrm{m}$ a $\mathrm{rk}$ 3.1. The process $X$ is not Markov under $\mathbf{Q}$. However, since $M_{t}^{\xi}$ is a function of $S_{t}$ and $X_{t}$, the pair $(X, S)$ is Markov under $\mathbf{Q}$.

Proposition 3.4. The Lévy system of $X$ under $\mathbf{Q}$ is given by

$$
\left(1-\frac{x \xi\left(S_{t}\right)}{M_{t}^{\xi}}\right) \nu(d x) d t
$$

P r o o f. Let $H$ be a positive, bounded predictable process, and let $f: \mathbf{R} \times \mathbf{R} \rightarrow \mathbf{R}_{+}$be a measurable nonnegative function such that $\int(1 \vee|y|) \times$ $f(x, x+y) \nu(d y)<\infty$ for all $x$. Denote by $A_{t}$ the process

$$
A_{t}=\sum_{s \leqslant t} H_{s} f\left(X_{s-}, X_{s}\right) .
$$

Let now $A^{c}$ be the dual predictable projection of $A$ under $\mathbf{P} ; A^{c}$ is given by

$$
A_{t}^{c}=\int_{0}^{t} d s H_{s} \int \nu(d x) f\left(X_{s}, X_{s}+x\right)
$$

and the compensated process $\widetilde{A}=A-A^{c}$ is a martingale. Then,

$$
\mathbf{Q}\left(A_{t}\right)=\mathbf{E}\left(M_{t}^{\xi} A_{t}\right)=\mathbf{E}\left(M_{t}^{\xi} \widetilde{A}_{t}\right)+\mathbf{E}\left(M_{t}^{\xi} A_{t}^{c}\right) .
$$

We have

$$
M_{t}^{\xi} A_{t}^{c}=\int_{0}^{t} A_{s}^{c} d M_{s}^{\xi}+\int_{0}^{t} M_{s}^{\xi} d A_{s}^{c}
$$

so that

$$
\mathbf{E}\left(M_{t}^{\xi} A_{t}^{c}\right)=\int_{0}^{t} d s \mathbf{E}\left(M_{s}^{\xi} H_{s} \int \nu(d x) f\left(X_{s}, X_{s}+x\right)\right) .
$$

On the other hand,

$$
\mathbf{E}\left(M_{t}^{\xi} \widetilde{A}_{t}\right)=\mathbf{E}\left[M^{\xi}, \widetilde{A}\right]_{t} .
$$

Since $\widetilde{A}$ is purely discontinuous, we have

$$
\left[M^{\xi}, \widetilde{A}\right]_{t}=\sum_{s \leqslant t} \Delta M_{s}^{\xi} \Delta \widetilde{A}_{s}=-\sum_{s \leqslant t} \Delta X_{s} \xi\left(S_{s}\right) H_{s} f\left(X_{s-}, X_{s}\right)
$$


and therefore

$$
\mathbf{E}\left(M_{t}^{\xi} \widetilde{A}_{t}\right)=-\mathbf{E}\left(\int_{0}^{t} d s H_{s} \xi\left(S_{s}\right) \int \nu(d x) x f\left(X_{s}, X_{s}+x\right)\right) .
$$

Putting things together, we obtain

$$
\begin{aligned}
\mathbf{Q}\left(A_{t}\right)= & \int_{0}^{t} d s \mathbf{E}\left(M_{s}^{\xi} H_{s} \int \nu(d x) f\left(X_{s}, X_{s}+x\right)\right. \\
& \left.-H_{s} \xi\left(S_{s}\right) \int \nu(d x) x f\left(X_{s}, X_{s}+x\right)\right) \\
= & \mathbf{Q}\left(\int_{0}^{t} d s H_{s} \int \nu(d x)\left(1-x \frac{g\left(S_{s}\right)}{M_{s}^{\xi}}\right) f\left(X_{s}, X_{s}+x\right)\right)
\end{aligned}
$$

which proves our claim.

$\mathrm{R}$ e $\mathrm{m}$ a $\mathrm{rk}$ 3.2. All the results of this section are valid if we start from a law $\mathbf{P}_{x}$ instead of $\mathbf{P}$.

$\mathrm{R}$ e $\mathrm{mark}$ 3.3. It is interesting to note that Assumption 3.1 is the condition under which the arcsine laws can be established for $X$, see [4].

4. Penalization and generalized Kennedy martingales. We are now interested in penalizations of the law $\mathbf{P}$ involving both $X_{t}$ and $S_{t}$. We first recall a family of martingales associated to $X$.

In this section, we assume that $\psi$ satisfies the following conditions.

Assumption 4.1.

$(\mathrm{K}): \quad \lambda_{0}:=\inf \{\lambda: \psi(\lambda)<\infty\}<0$ and $\beta^{*}:=\psi\left(\lambda_{0}+\right)>0$.

Under these conditions, for each $\beta \in\left(0, \beta^{*}\right)$, the equation $\psi(\lambda)=\beta$ has two distinct roots which we denote by $\gamma^{+}$and $\gamma^{-}$, with $\gamma^{-}<0<\gamma^{+}$. Set

$$
\eta=\eta(\beta)=\frac{1}{2}\left(\gamma^{+}+\gamma^{-}\right), \quad \delta=\delta(\beta)=\frac{1}{2}\left(\gamma^{+}-\gamma^{-}\right) .
$$

For $\alpha \geqslant 0$, let $h$ be the function

$$
h(x)=e^{-\eta x}((\alpha+\eta) \operatorname{sh},(\delta x)+\delta \operatorname{ch}(\delta x)) .
$$

Then the process

$$
e^{-\alpha S_{t}-\beta t} h\left(S_{t}-X_{t}\right)
$$

is a local martingale, called the generalized Kennedy martingale (see [9]).

We denote by $\mathbf{P}^{( \pm)}$the Esscher transform of $\mathbf{P}$ with parameter $\gamma^{ \pm}$:

$$
\left.\mathbf{P}^{( \pm)}\right|_{\mathscr{F}_{t}}=\left.e^{\gamma^{ \pm} X_{t}-\beta t} \mathbf{P}\right|_{\mathscr{F}_{t}} .
$$

Then, under $\mathbf{P}^{( \pm)}, X$ is a Lévy process with Laplace exponent

$$
\psi^{( \pm)}(\lambda)=\psi\left(\lambda+\gamma^{ \pm}\right)-\beta
$$


whose inverse is

$$
\Phi^{( \pm)}(q)=\Phi(q+\beta)-\gamma^{ \pm} .
$$

Note that $X$ drifts to $-\infty$ (respectively, $+\infty$ ) under $\mathbf{P}^{(-)}$(respectively, $\mathbf{P}^{(+)}$); in fact these two probability measures are related by the absolute continuity relationship:

$$
\left.\mathbf{P}^{(+)}\right|_{\mathscr{F}_{t}}=\left.e^{2 \delta X_{t}} \mathbf{P}^{(-)}\right|_{\mathscr{F}_{t}}
$$

and $\mathbf{P}^{(+)}$is the measure $\mathbf{P}^{(-)} \ll$ conditioned to drift to $+\infty \gg$ (see [3], [4]).

Fix $\beta \in\left(0, \beta^{*}\right)$ and let $\xi: \mathbf{R}_{+} \rightarrow \mathbf{R}_{+}$be a nonnegative measurable function; we set

$$
\xi_{\beta}(x)=e^{-\eta x} \xi(x)
$$

and assume that $\xi_{\beta}$ is integrable. We define a function $\Xi_{\beta}$ by

$$
\Xi_{\beta}(x)=\xi_{\beta}(x)-\delta e^{\delta x} \int_{x}^{\infty} e^{-\delta y} \xi_{\beta}(y) d y
$$

and let

$$
\bar{\Xi}_{\beta}(x)=\int_{x}^{\infty} \Xi_{\beta}(y) d y=e^{\delta x} \int_{x}^{\infty} e^{-\delta y} \xi_{\beta}(y) d y .
$$

We shall assume throughout this section that

$$
\mathbf{E} e^{-\gamma^{-}\left(S_{t}-X_{t}\right)}<\infty
$$

for all $t$. Our goal is the study of $\lim _{t \rightarrow \infty} \mathbf{Q}_{t}$, where

$$
\mathbf{Q}_{t}(\Lambda)=\frac{\mathbf{E}\left[\xi\left(S_{t}\right) e^{-\gamma^{-}\left(S_{t}-X_{t}\right)} 1_{\Lambda}\right]}{\mathbf{E}\left[\xi\left(S_{t}\right) e^{-\gamma^{-}\left(S_{t}-X_{t}\right)}\right]}, \quad \Lambda \in \mathscr{F}_{u}, \quad u \leqslant t
$$

We start with the following analogue of Lemma 3.1.

Lemma 4.1. For $x \in \mathbf{R}$ and $s \geqslant 0 \vee x$,

$$
e^{-\beta t} \mathbf{E}\left[\xi\left(s \vee\left(x+S_{t}\right)\right) e^{-\gamma^{-}\left(s \vee\left(x+S_{t}\right)-x-X_{t}\right)}\right] \sim \rho_{\beta}(x, s), \quad t \rightarrow \infty,
$$

where

$$
\rho_{\beta}(x, s)=2 e^{\eta x}\left[\Xi_{\beta}(s) \operatorname{sh},(\delta(s-x))+\delta \bar{\Xi}_{\beta}(s) \operatorname{ch}(\delta(s-x))\right] .
$$

P r o o f. First note that

$$
\begin{aligned}
e^{-\beta t} \mathbf{E}\left[\xi\left(s \vee\left(x+S_{t}\right)\right) e^{-\gamma^{-}\left(s \vee\left(x+S_{t}\right)-x-X_{t}\right)}\right] \\
=\mathbf{E}^{(-)}\left(\xi\left(s \vee\left(x+S_{t}\right)\right) e^{-\gamma^{-}\left((s-x) \vee S_{t}\right)}\right) .
\end{aligned}
$$


Let $\tau$ be an exponential variable with parameter $q>0$, independent of $X$. We have

$$
\begin{aligned}
\mathbf{E}^{(-)}\left(\xi\left(s \vee\left(x+S_{\tau}\right)\right) e^{-\gamma^{-}\left((s-x) \vee S_{\tau}\right)}\right) & \\
= & \xi(s) e^{-\gamma^{-}(s-x)} \mathbf{P}^{(-)}\left(S_{\tau}<s-x\right)+\mathbf{E}^{(-)}\left(\xi\left(x+S_{\tau}\right) e^{-\gamma^{-} S_{\tau}}\right) \\
= & \xi(s) e^{-\gamma^{-}(s-x)}\left(1-e^{-\Phi^{(-)}(q)(s-x)}\right) \\
& +\int_{s-x}^{\infty} \xi(x+y) e^{-\gamma^{-} y} \Phi^{(-)}(q) e^{-\Phi^{(-)}(q) y} d y \\
= & \xi(s) e^{-\gamma^{-}(s-x)}\left(1-e^{-\Phi^{(-)}(q)(s-x)}\right) \\
& +\Phi^{(-)}(q) e^{\left(\gamma^{-}+\Phi^{(-)}(q)\right) x} \int_{s}^{\infty} \xi(y) e^{-\left(\gamma^{-}+\Phi^{(-)}(q)\right) y} d y .
\end{aligned}
$$

Since $\Phi^{(-)}(0)=\Phi(\beta)-\gamma^{-}=\gamma^{+}-\gamma^{-}=2 \delta$, we have as $q \downarrow 0$ :

$$
\begin{aligned}
\mathbf{E}^{(-)} & \left(\xi\left(s \vee\left(x+S_{\tau}\right)\right) e^{-\gamma^{-}\left((s-x) \vee S_{\tau}\right)}\right) \\
& \sim \xi(s) e^{-\gamma^{-}(s-x)}\left(1-e^{-2 \delta(s-x)}\right)+2 \delta e^{\gamma^{+} x} \int_{s}^{\infty} \xi(y) e^{-\gamma^{+} y} d y \\
& =\xi(s)\left(e^{-(\eta-\delta)(s-x)}-e^{-(\eta+\delta)(s-x)}\right)+2 \delta e^{(\eta+\delta) x} \int_{s}^{\infty} \xi(y) e^{-(\eta+\delta) y} d y .
\end{aligned}
$$

Straightforward computations show that the foregoing is equal to the function $\rho_{\beta}$ defined in (4.6). The equivalence (4.5) follows from a Tauberian theorem. Lemma 4.1 is proved.

We can now state the following regarding the existence of $\lim _{t \rightarrow \infty} \mathbf{Q}_{t}^{\beta}$.

Theorem 4.1. For $u \geqslant 0$ and $\Lambda \in \mathscr{F}_{u}$, the limit

$$
\lim _{t \rightarrow \infty} \frac{\mathbf{E}\left[\xi\left(S_{t}\right) e^{-\gamma^{-}\left(S_{t}-X_{t}\right)} 1_{\Lambda}\right]}{\mathbf{E}\left[\xi\left(S_{t}\right) e^{-\gamma^{-}\left(S_{t}-X_{t}\right)}\right]}
$$

exists and is equal to

$$
\mathbf{E}\left[M_{u}^{\beta, \xi} 1_{\Lambda}\right]
$$

where

$$
M_{t}^{\beta, \xi}=e^{-\beta t} \frac{\rho_{\beta}\left(X_{u}, S_{u}\right)}{\rho_{\beta}(0,0)} .
$$

$\mathrm{P}$ r o o f. For $t>u$, we have

$$
\frac{\mathbf{E}\left[\xi\left(S_{t}\right) e^{-\gamma^{-}\left(S_{t}-X_{t}\right)} 1_{\Lambda}\right]}{\mathbf{E}\left[\xi\left(S_{t}\right) e^{-\gamma^{-}\left(S_{t}-X_{t}\right)}\right]}=\mathbf{E}\left(1_{\Lambda} \frac{h_{t}\left(X_{u}, S_{u}\right)}{h_{t}(0,0)}\right)
$$

where $h_{t}(x, s)=\mathbf{E}\left(\xi\left(S_{t}\right) e^{-\gamma^{-}\left(S_{t}-X_{t}\right)} \mid \mathscr{F}_{u}, X_{u}=x, S_{u}=s\right)$. By Lemma 4.1,

$$
\frac{h_{t}\left(X_{u}, S_{u}\right)}{h_{t}(0,0)} \sim e^{-\beta u} \frac{\rho_{\beta}\left(X_{u}, S_{u}\right)}{\rho_{\beta}(0,0)}, \quad t \rightarrow \infty,
$$

and the result follows. 
$\mathrm{R}$ e $\mathrm{m}$ a r k 4.1. One may ask whether it would be possible to choose $\gamma^{+}$instead of $\gamma^{-}$to define a penalization function, that is, to study the probability measures

$$
Q_{t}^{+}(\Lambda)=\frac{\mathbf{E}\left(1_{\Lambda} \xi\left(S_{t}\right) e^{\gamma^{+}\left(S_{t}-X_{t}\right)}\right)}{\mathbf{E}\left(\xi\left(S_{t}\right) e^{\gamma^{+}\left(S_{t}-X_{t}\right)}\right)}, \quad \Lambda \in \mathscr{F}_{u}, \quad u \leqslant t
$$

as $t \rightarrow \infty$. However the technique employed here would not apply because $\Phi^{(+)}(0)=0$, which makes it impossible to use the Tauberian theorem as it is in Lemma 4.1 .

It is easy to check that $M^{\beta, \xi}$ is a local martingale (just apply Itô's formula), that coincides with the Kennedy (local) martingale with parameters $\alpha, \beta$ if one chooses $\xi(x)=e^{-\alpha x}$. From now on, we assume that it is a true martingale.

Ex a m p le 4.1. Assume that $\xi$ is bounded and that $e^{\gamma^{+} S_{t}}$ and $e^{-\gamma^{-}\left(S_{t}-I_{t}\right)}$ are integrable. Then, $M^{\beta, \xi}$ is a class (D) local martingale, hence a true martingale.

From now on, to simplify the notation, we assume that $\xi$ is normalized in such a way that $\rho_{\beta}(0,0)=1$, that is:

$$
\int_{0}^{\infty} \xi_{\beta}(x) e^{-\delta x} d x=\frac{1}{2 \delta} .
$$

We denote by $\mathbf{Q}^{\beta, \xi}$ the probability measure on $\Omega$ such that for all $u \geqslant 0$

$$
\left.\mathbf{Q}^{\beta, \xi}\right|_{\mathscr{F}_{u}}=\left.M_{u}^{\beta, \xi} \mathbf{P}\right|_{\mathscr{F}_{u}} .
$$

We now study the law $\mathbf{Q}^{\beta, \xi}$ along the same lines as in the previous section. First of all, we prove the following proposition.

Proposition 4.1. Under $\mathbf{Q}^{\beta, \xi}, S_{\infty}$ is finite a.s. Moreover,

$$
\mathbf{Q}^{\beta, \xi}\left[S_{\infty}>x\right]=2 \delta e^{-\delta x} \bar{\Xi}_{\beta}(x) .
$$

$\mathrm{P}$ r o o f. It is completely similar to the proof of Proposition 3.2. Let $\tau$ be an exponential variable with parameter $q>0$, independent of $X$. It will be convenient to use the following expression of $\rho_{\beta}$ :

$$
\rho_{\beta}(x, s)=\xi(s)\left(e^{-\gamma^{-}(s-x)}-e^{-\gamma^{+}(s-x)}\right)+2 \delta e^{\gamma^{+} x} \int_{s}^{\infty} \xi(y) e^{-\gamma^{+} y} d y .
$$

We have

$$
\begin{aligned}
& \mathbf{Q}^{\beta}\left(S_{\tau}>x\right)= \mathbf{E}\left(e^{-\beta \tau} \rho_{\beta}\left(X_{\tau}, S_{\tau}\right) 1_{S_{\tau}>x}\right) \\
&= \mathbf{E}\left(1 _ { S _ { \tau } > x } e ^ { - \beta \tau } \left\{\xi\left(S_{\tau}\right)\left(e^{-\gamma^{-}\left(S_{\tau}-X_{\tau}\right)}-e^{-\gamma^{+}\left(S_{\tau}-X_{\tau}\right)}\right)\right.\right. \\
&\left.\left.\quad+2 \delta e^{\gamma^{+} X_{\tau}} \int_{S_{\tau}}^{\infty} \xi(y) e^{-\gamma^{+} y} d y\right\}\right) \\
&=\mathbf{E}^{(+)}\left(1_{S_{\tau}>x}\left\{2 \delta \int_{S_{\tau}}^{\infty} \xi(y) e^{-\gamma^{+} y} d y-\xi\left(S_{\tau}\right) e^{-\gamma^{+} S_{\tau}}\right\}\right) \\
&+\mathbf{E}^{(-)}\left(1_{S_{\tau}>x} \xi\left(S_{\tau}\right) e^{-\gamma-S_{\tau}}\right) .
\end{aligned}
$$


As to the former term, we have, on the one hand,

$$
\begin{aligned}
\mathbf{E}^{(+)}\left(1_{S_{\tau}>x} \int_{S_{\tau}}^{\infty} \xi(y) e^{-\gamma^{+} y} d y\right) & =\Phi^{+}(q) \int_{x}^{\infty}\left(e^{-\Phi^{+}(q) s} \int_{s}^{\infty} \xi(y) e^{-\gamma^{+} y} d y\right) d s \\
& =\int_{x}^{\infty} \xi(y)\left(e^{-\Phi^{+}(q) x}-e^{-\Phi^{+}(q) y}\right) e^{-\gamma^{+} y} d y
\end{aligned}
$$

Since $\xi(x) e^{-\gamma^{+} x}=\xi_{\beta}(x) e^{-\delta x}$ is integrable and $\Phi^{+}(q) \rightarrow 0$ as $q \rightarrow 0$, we have

$$
\mathbf{E}^{(+)}\left(1_{S_{\tau}>x} \int_{S_{\tau}}^{\infty} \xi(y) e^{-\gamma^{+} y} d y\right) \longrightarrow 0, \quad q \rightarrow 0 .
$$

On the other hand,

$$
\mathbf{E}^{(+)}\left(1_{S_{\tau}>x} \xi\left(S_{\tau}\right) e^{-\gamma^{+} S_{\tau}}\right)=\Phi^{+}(q) \int_{x}^{\infty} e^{-\Phi^{+}(q) s} \xi(s) e^{-\gamma^{+} s} d s
$$

and this also tends to 0 as $q \rightarrow 0$. Lastly,

$$
\begin{aligned}
\mathbf{E}^{(-)}\left(1_{S_{\tau}>x} \xi\left(S_{\tau}\right) e^{-\gamma^{-} S_{\tau}}\right) & =\Phi^{-}(q) \int_{x}^{\infty} e^{-\left(\Phi^{-}(q)+\gamma^{-}\right) s} \xi(s) d s \\
& \rightarrow 2 \delta \int_{x}^{\infty} \xi(s) e^{-(\eta+\delta) s} d s=2 \delta e^{-\delta x} \bar{\Xi}_{\beta}(x), \quad q \rightarrow 0 .
\end{aligned}
$$

Proposition 4.1 is proved.

We now give a description of the disintegration of $\mathbf{Q}^{\beta, \xi}$ with respect to $S_{\infty}$, in the form of a path decomposition of $X$ under $\mathbf{Q}^{\beta, \xi}$. Recall that $g$ denotes the last time $X$ attains its overall supremum, $g=\sup \left\{t: X_{t}=S_{\infty}\right\}$.

Theorem 4.2. Under $\mathbf{Q}^{\beta, \xi}$, conditionally on $S_{\infty}=y$, the processes $\left(X_{t}, t<g\right)$ and $\left(X_{g+t}, t \geqslant 0\right)$ are independent. Moreover,

(a) The law of $\left(X_{t}, t<g\right)$ under $\mathbf{Q}^{\beta, \xi}\left(\cdot \mid S_{\infty}=y\right)$ is the same as the law of $\left(X_{t}, t<T_{y}\right)$ under $\mathbf{P}^{(+)}$;

(b) for every measurable, bounded functional $F$, we have

$$
\mathbf{Q}^{\beta, \xi}\left[F\left(X_{g+t}, t \geqslant 0\right) \mid S_{\infty}=y\right]=\mathbf{E}^{(-) \downarrow}\left[F\left(y+X_{t}, t \geqslant 0\right)\right] .
$$

This theorem could be proved using the same technique as in Theorem 3.2, however there is a simpler way that hinges on [7], [3] and the following Proposition 4.2.

Let $\widetilde{M}^{\beta, \xi}$ be the process defined by

$$
\widetilde{M}_{t}^{\beta, \xi}=e^{-\gamma^{-} X_{t}} \rho_{\beta}\left(X_{t}, S_{t}\right) .
$$

Proposition 4.2. On $(\Omega, \mathscr{F})$, the following absolute continuity relation takes place:

$$
\mathbf{Q}^{\beta, \xi}=\widetilde{M}_{\infty}^{\beta, \xi} \mathbf{P}^{(-)},
$$

where

$$
\widetilde{M}_{\infty}^{\beta, \xi}=2 e^{\delta S_{\infty}}\left(\Xi_{\beta}\left(S_{\infty}\right)+\delta \bar{\Xi}_{\beta}\left(S_{\infty}\right)\right)=2 e^{\delta S_{\infty}} \xi_{\beta}\left(S_{\infty}\right) .
$$


P r o o f. Since $e^{-\beta t} \rho_{\beta}\left(X_{t}, S_{t}\right)$ is a $\mathbf{P}$-martingale, $\widetilde{M}^{\beta, \xi}$ is a $\mathbf{P}^{(-)}$martingale. Since $X_{t} \rightarrow-\infty$ under $\mathbf{P}^{(-)}$, we have $\widetilde{M}_{t}^{\beta, \xi} \rightarrow \widetilde{M}_{\infty}^{\beta, \xi} \mathbf{P}^{(-)}$-a.s.

Moreover under $\mathbf{P}^{(-)}, S_{\infty}$ has an exponential distribution with parameter $2 \delta$. It follows that $\widetilde{M}_{\infty}^{\beta, \xi} \in L^{1}\left(\mathbf{P}^{(-)}\right)$and it is easily checked that $\widetilde{M}_{t}^{\beta, \xi}=\mathbf{E}^{(-)}\left(\widetilde{M}_{\infty}^{\beta, \xi} \mid \mathscr{F}_{t}\right)$ for all $t$.

Let $Q$ be the probability measure on $(\Omega, \mathscr{F})$, defined by

$$
Q=\widetilde{M}_{\infty}^{\beta, \xi} \mathbf{P}^{(-)} .
$$

Then for all $t>0$, we have

$$
\left.Q\right|_{\mathscr{F}_{t}}=\left.\widetilde{M}_{t}^{\beta, \xi} \mathbf{P}^{(-)}\right|_{\mathscr{F}_{t}}=\left.e^{\eta X_{t}-\beta t} \rho_{\beta}\left(X_{t}, S_{t}\right) \mathbf{P}\right|_{\mathscr{F}_{t}}=\left.\mathbf{Q}^{\beta, \xi}\right|_{\mathscr{F}_{t}} .
$$

This implies that $Q=\mathbf{Q}^{\beta, \xi}$. Proposition 4.2 is proved.

By conditioning on $S_{\infty}$ in identity (4.11), we immediately obtain the following corollary, which expresses $\mathbf{Q}^{\beta, \xi}$ as a weak conditioning of $S_{\infty}$ under $\mathbf{P}^{(-)}$. Denote by $\sigma^{\beta, \xi}$ the density of $S_{\infty}$ under $\mathbf{Q}^{\beta, \xi}$ :

$$
\sigma^{\beta, \xi}(x)=2 \delta e^{-\delta x} \xi_{\beta}(x), \quad x \geqslant 0 .
$$

Corollary 4.1. We have

$$
\mathbf{Q}^{\beta, \xi}=\int_{0}^{\infty} \mathbf{P}^{(-)}\left(\cdot \mid S_{\infty}=y\right) \sigma^{\beta, \xi}(y) d y .
$$

Pr o of of $\mathrm{T}$ h e or e $\mathrm{m}$ 4.2. The results of [3], [7] can be summarized as follows:

(a) The processes $X^{g}$ and $\left(X_{g+t}-S_{\infty}, t \geqslant 0\right)$ are independent;

(b) the law of the post-maximum process $\vec{X}=\left(X_{g+t}-S_{\infty}, t \geqslant 0\right)$ is $\mathbf{P}^{(-) \downarrow}$;

(c) the law of $X^{g}$ under $\mathbf{P}^{(-)}$is the same as the law of $X^{T_{\tau}}$ under $\mathbf{P}^{(+)}$, where $\tau$ is and exponential variable with parameter $2 \delta$, independent of $X$.

Let $H$ and $K$ be two measurable, bounded functionals on $\Omega$. On the one hand, we deduce from the results just recalled that

$$
\mathbf{E}^{(-)}\left(H\left(X^{g}\right) K(\vec{X})\right)=\int_{0}^{\infty} \mathbf{E}^{(+)}\left(H\left(X^{T_{y}}\right)\right) \cdot 2 \delta e^{-2 \delta y} d y \mathbf{E}^{(-) \downarrow}(K) .
$$

On the other hand, since $S_{\infty}$ is exponentially distributed with parameter $2 \delta$,

$$
\mathbf{E}^{(-)}\left(H\left(X^{g}\right) K(\vec{X})\right)=\int_{0}^{\infty} 2 \delta e^{-2 \delta y} \mathbf{E}^{(-)}\left(H\left(X^{g}\right) K(\vec{X}) \mid S_{\infty}=y\right) d y .
$$

Therefore, under $\mathbf{P}^{(-)}\left(\cdot \mid S_{\infty}=y\right)$

(a) $X^{g}$ and $\vec{X}$ are independent;

(b) the law of $X^{g}$ is the same as the law of $X^{T_{y}}$ under $\mathbf{P}^{(+)}$;

(c) the law of $X_{g+t}-y$ is $\mathbf{P}^{(-) \downarrow}$. 
Thanks to (4.11) we obtain

$$
\begin{aligned}
& \mathbf{Q}^{\beta, \xi}\left(H\left(X^{g}\right) K\left(X_{g+t}, t \geqslant 0\right)\right) \\
& \quad=\int_{0}^{\infty} \sigma^{\beta, \xi}(y) \mathbf{E}^{(+)}\left(H\left(X^{T_{y}}\right)\right) \mathbf{E}^{(-) \downarrow}\left(K\left(y+X_{t}, t \geqslant 0\right)\right) d y
\end{aligned}
$$

and this concludes the proof.

We end this paper with the characterization of the jumps of the process $X$ under $\mathbf{Q}^{\beta, \xi}$, thanks to the following proposition.

Proposition 4.3. The Lévy system of $X$ under $\mathbf{Q}^{\beta, \xi}$ is given by

$$
\frac{\rho_{\beta}\left(X_{t}+z, S_{t}\right)}{\rho_{\beta}\left(X_{t}, S_{t}\right)} \nu(d z) d t
$$

P r o o f. Let $H$ be a bounded predictable process and $f: \mathbf{R} \times \mathbf{R} \rightarrow \mathbf{R}$ a bounded Borel function such that $\int(1 \vee|y|) f(x, x+y) \nu(d y)<\infty$ for all $x$; set

$$
A_{t}=\sum_{s \leqslant t} H_{s} f\left(X_{s-}, X_{s}\right) 1_{\Delta X_{s} \neq 0}
$$

We have

$$
\mathbf{Q}^{\beta, \xi}\left(A_{t}\right)=\mathbf{E}\left(M_{t}^{\beta, \xi} \widetilde{A}_{t}\right)+\mathbf{E}\left(M_{t}^{\beta, \xi} A_{t}^{c}\right),
$$

where $A^{c}$ is the predictable compensator of $A$ :

$$
A_{t}^{c}=\int_{0}^{t} H_{s} \int f\left(X_{s}, X_{s}+x\right) \nu(d x) d s
$$

and $\widetilde{A}_{t}=A_{t}-A_{t}^{c}$ is a martingale. Proceeding as in the proof of Proposition 3.4 , we obtain, on the one hand

$$
\begin{gathered}
\mathbf{E}\left(M_{t}^{\beta, \xi} \widetilde{A}_{t}\right)=\mathbf{E}\left(\int _ { 0 } ^ { t } H _ { s } d s \int f ( X _ { s } , X _ { s } + x ) e ^ { - \beta s } \left(\rho_{\beta}\left(X_{s}+x, S_{s}\right)\right.\right. \\
\left.\left.-\rho_{\beta}\left(X_{s}, S_{s}\right)\right) \nu(d x)\right)
\end{gathered}
$$

and on the other hand

$$
\mathbf{E}\left(M_{t}^{\beta, \xi} A_{t}^{c}\right)=\mathbf{E}\left(\int_{0}^{t} H_{s} d s \int f\left(X_{s}, X_{s}+x\right) M_{s}^{\beta, \xi} \nu(d x)\right) .
$$

Thus,

$$
\begin{aligned}
& \mathbf{Q}^{\beta, \xi}\left(A_{t}\right) \\
& =\mathbf{E}\left(\int_{0}^{t} H_{s} d s \int f\left(X_{s}, X_{s}+x\right) e^{-\beta s}\left(\rho_{\beta}\left(X_{s}+x, S_{s}\right)-\rho_{\beta}\left(X_{s}, S_{s}\right)\right) \nu(d x)\right) \\
& \quad+\mathbf{E}\left(\int_{0}^{t} H_{s} d s \int f\left(X_{s}, X_{s}+x\right) M_{s}^{\beta, \xi} \nu(d x)\right)
\end{aligned}
$$




$$
\begin{gathered}
=\mathbf{E}\left(\int _ { 0 } ^ { t } H _ { s } d s \int \nu ( d x ) f ( X _ { s } , X _ { s } + x ) \left[e^{-\beta s}\left(\rho_{\beta}\left(X_{s}+x, S_{s}\right)-\rho_{\beta}\left(X_{s}, S_{s}\right)\right)\right.\right. \\
\left.\left.+e^{-\beta s} \rho_{\beta}\left(X_{s}, S_{s}\right)\right]\right) \\
=\mathbf{E}\left(\int_{0}^{t} H_{s} d s \int \nu(d x) f\left(X_{s}, X_{s}+x\right) M_{s}^{\beta, \xi} \frac{\rho_{\beta}\left(X_{s}+x, S_{s}\right)}{\rho_{\beta}\left(X_{s}, S_{s}\right)}\right) \\
=\mathbf{Q}^{\beta, \xi}\left(\int_{0}^{t} H_{s} d s \int \nu(d x) f\left(X_{s}, X_{s}+x\right) \frac{\rho_{\beta}\left(X_{s}+x, S_{s}\right)}{\rho_{\beta}\left(X_{s}, S_{s}\right)}\right) .
\end{gathered}
$$

\section{REFERENCES}

1. Baudoin F. Conditioned stochastic differential equations: Theory, examples and application to finance. - Stochastic Process. Appl., 2002, v. 100, p. 109-145.

2. Baudoin F., Nguyen-Ngoc L. The financial value of a weak information in a financial market. - Finance Stoch., 2004, v. 8, № 3, p. 415-435.

3. Bertoin J. Sur la décomposition de la trajectoire d'un processus de Lévy spectralement positif en son infimum. - Ann. Inst. H. Poincaré, 1991, v. 27, № 4, p. 537-547.

4. Bertoin J. Lévy Processes. Cambridge: Cambridge Univ. Press, 1996, 265 p.

5. Chaumont L. Sur certains processus de Lévy conditionnés à rester positifs. - Stochastics Stochastics Rep., 1994, v. 47, № 1-2, p. 1-20.

6. Феллер В. Введение в теорию вероятностей и ее приложения, т. 2. М.: Мир, 1984, $752 \mathrm{c}$.

7. Millar P. W. Zero-one laws and the minimum of a Markov process. - Trans. Amer. Math. Soc., 1977, v. 226, p. 365-391.

8. Nguyen-Ngoc L. Weak conditioning for jump processes. Technical report № 851. Paris: Universités de Paris 6 \& Paris 7, 2003.

9. Nguyen-Ngoc L., Yor M. Some martingales associated to reflected Lévy processes. Lecture Notes in Math., 2005, v. 1857, p. 42-69.

10. Roynette B., Vallois P., Yor M. Limiting laws associated with Brownian motion perturbated by normalized exponential weights. - C. R. Math. Acad. Sci. Paris, 2003 , v. 337 , № 10 , p. 667-673.

11. Roynette B., Vallois P., Yor M. Limiting laws associated with Brownian motion perturbed by its maximum, minimum and local time. II. - Studia Sci. Math. Hungar., 2006, v. 43, № 3, p. 295-360.

12. Roynette B., Vallois P., Yor M. Pénalisations et quelques extensions du théorème de Pitman, relatives au mouvement brownien et à son maximum unilatère. - Lecture Notes in Math., 2006, v. 1874, p. 305-336. 